\title{
MedTag: A Collection of Biomedical Annotations
}

\author{
L.H. Smith ${ }^{\dagger}$, L. Tanabe ${ }^{\dagger}$, T. Rindflesch ${ }^{\ddagger}$, W.J. Wilbur ${ }^{\dagger}$ \\ ${ }^{\dagger}$ National Center for Biotechnology Information \\ ${ }^{\ddagger}$ Lister Hill National Center for Biomedical Communications \\ NLM, NIH, 8600 Rockville Pike, Bethesda, MD 20894 \\ \{lsmith,tanabe,wilbur\}@ncbi.nlm.nih.gov \\ rindflesch@nlm.nih.gov
}

\begin{abstract}
We present a database of annotated biomedical text corpora merged into a portable data structure with uniform conventions. MedTag combines three corpora, MedPost, ABGene and GENETAG, within a common relational database data model. The GENETAG corpus has been modified to reflect new definitions of genes and proteins. The MedPost corpus has been updated to include 1,000 additional sentences from the clinical medicine domain. All data have been updated with original MEDLINE text excerpts, PubMed identifiers, and tokenization independence to facilitate data accuracy, consistency and usability.

The data are available in flat files along with software to facilitate loading the data into a relational SQL database from ftp://ftp.ncbi.nlm.nih.gov/pub/lsmith /MedTag/medtag.tar.gz.
\end{abstract}

\section{Introduction}

Annotated text corpora are used in modern computational linguistics research and development to finetune computer algorithms for analyzing and classifying texts and textual components. Two important factors for useful text corpora are 1) accuracy and consistency of the annotations, and 2) usability of the data. We have recently updated the text corpora we use in our research with respect to these criteria.
Three different corpora were combined. The ABGene corpus consists of over 4000 sentences annotated with gene and protein named entities. It was originally used to train the ABGene tagger to recognize gene/protein names in MEDLINE records, and recall and precision rates in the lower 70 percentile range were achieved (Tanabe and Wilbur, 2002). The MedPost corpus consists of 6700 sentences, and is annotated with parts of speech, and gerund arguments. The MedPost tagger was trained on 3700 of these sentences and achieved an accuracy of $97.4 \%$ on the remaining sentences (Smith et. al., 2004). The GENETAG corpus for gene/protein named entity identification, consists of 20000 sentences and was used in the BioCreative 2004 Workshop (Yeh et. al., 2005; Tanabe et. al., 2005) (only 15000 sentences are currently released, the remaining 5000 are being retained for possible use in a future workshop). Training on a portion of the data, the top performing systems achieved recall and precision rates in the lower 80 percentile range. Because of the scarcity of good annotated data in the realm of biomedicine, and because good performance has been obtained using this data, we feel there is utility in presenting it to a wider audience.

All of the MedTag corpora are based on MEDLINE abstracts. However, they were queried at different times, and used different (but similar) algorithms to perform tokenization and sentence segmentation. The original annotations were assigned to tokens, or sequences of tokens, and extensively reviewed by the authors at different times for the different research projects.

The main goals in combining and updating these 


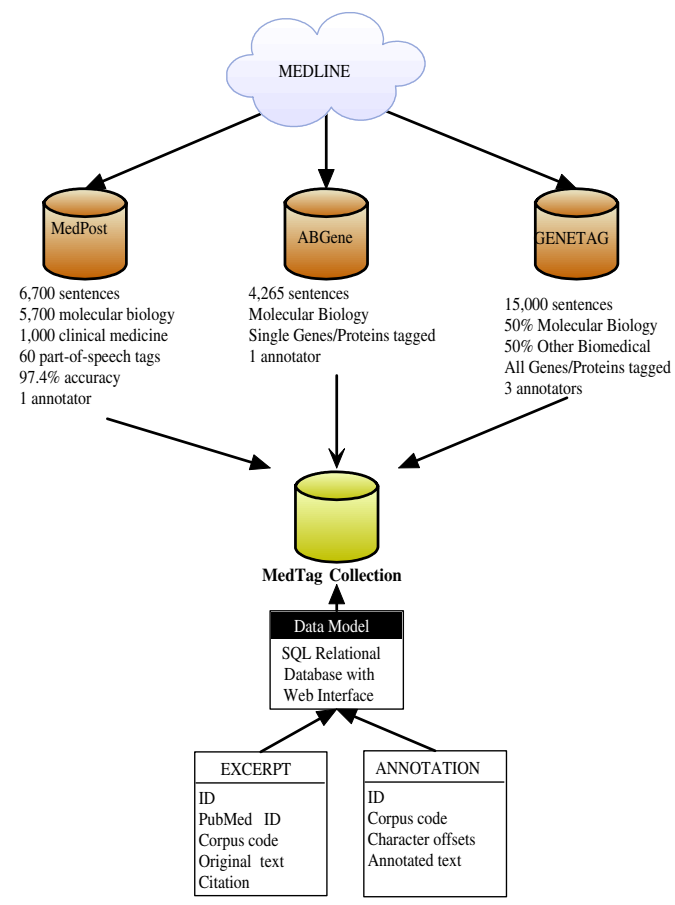

Figure 1: Component corpora, common data model and main record types of the MedTag collection.

corpora into a single corpus were to

1. update the text for all corpora to that currently found in MEDLINE, storing a correct citation and the original, untokenized text for each excerpt

2. eliminate tokenization dependence

3. put all text and annotations into a common database format

4. provide programs to convert from the new corpus format to the data formats used in previous research

\section{Merging the Corpora}

We describe what was done to merge the original corpora, locating original sources and modifying the text where needed. An overview is given in Figure 1. Some basic statistics are given in Table 1.

\subsection{Identifying Source Data}

The original data of the three corpora were assembled and the text was used to search MEDLINE to

\begin{tabular}{cccc} 
Corpus & sentences & tokens & most frequent tag \\
\hline GENETAG-05 & 15,000 & 418,246 & insulin_GENE(112) \\
MedPost & 6,700 & 181,626 & the_DD $(8,507)$ \\
AbGene & 4,265 & 123,208 & cyclin_GENE $(165)$
\end{tabular}

\begin{tabular}{cccccc}
\multicolumn{6}{c}{ MedPost } \\
Adj & Adv & Aux & Noun & Punct & Verb \\
\hline 14,648 & 4,553 & 56,262 & 60,732 & 21,806 & 23,625 \\
& & & & \\
GENETAG-05 & & \\
& \multicolumn{3}{c}{ GENE } & ALTGENE & \\
\cline { 2 - 3 } & 24,562 & 19,216 &
\end{tabular}

\begin{tabular}{cc}
\multicolumn{2}{c}{ ABGene } \\
GENE & ALTGENE \\
\hline 8,185 & 0
\end{tabular}

Table 1: MedTag Corpora. GENE = gene and protein names, ALTGENE = acceptable alternatives for gene and protein names. MedPost tagset contains 60 parts of speech which have been binned here for brevity.

find the closest match. An exact or near exact match was found for all but a few excerpts. For only a few excerpts, the MEDLINE record from which the excerpt was originally taken had been removed or modified and an alternative sentence was selected. Thus, each excerpt in the database is taken from a MEDLINE record as it existed at one time in 2004. In order to preserve the reference for future work, the PubMed ID and citation data were also retrieved and stored with each excerpt. Each excerpt in the current database roughly corresponds to a sentence, although the procedure that extracted the sentence is not specified.

\subsection{Eliminating Tokenization Dependence}

In the original ABGene and GENETAG corpora, the gene and protein phrases were specified by the tokens contained in the phrase, and this introduced a dependence on the tokenization algorithm. This created problems for researchers who wished to use a different tokenization. To overcome this dependence, we developed an alternative way of specify- 
ing phrases. Given the original text of an excerpt, the number of non-whitespace characters to the start of the phrase does not depend on the tokenization. Therefore, all annotations now refer to the first and last character of the phrase that is annotated. For example the protein serum $\mathrm{LH}$ in the excerpt

There was no correlation between serum $L H$ and chronological or bone age in this age group, which suggests that the correlation found is not due to age-related parallel phenomena.

is specified as characters 28 to 34 (the first character is 0 ).

\subsection{Data Model}

There are two main record types in the database, EXCERPT and ANNOTATION. Each EXCERPT record stores an identifier and the original corpus code (abgene, medpost, and genetag) as well as subcorpus codes that were defined in the original corpora. The original text, as it was obtained from MEDLINE, is also stored, and a human readable citation to the article containing the reference.

Each ANNOTATION record contains a reference to the excerpt (by identifier and corpus), the character offset of the first and last characters of the phrase being annotated (only non-whitespace characters are counted, starting with 0 ), and the corresponding annotation. The annotated text is stored for convenience, though it can be obtained from the corresponding excerpt record by counting nonwhitespace characters.

The data is provided as an ASCII file in a standard format that can be read and loaded into a relational database. Each record in the file begins with a line of the form $>>$ table_name where table_name is the name of the table for that record. Following the table name is a series of lines with the form field: value where field is the name of the field and value is the value stored in that field.

Scripts are provided for loading the data into a relational database, such as mysql or ORACLE. SQL queries can then be applied to retrieve excerpts and annotations satisfying any desired condition. For example, here is an SQL query to retrieve excerpts from the MedPost corpus containing the token $p 53$ and signaling or signalling

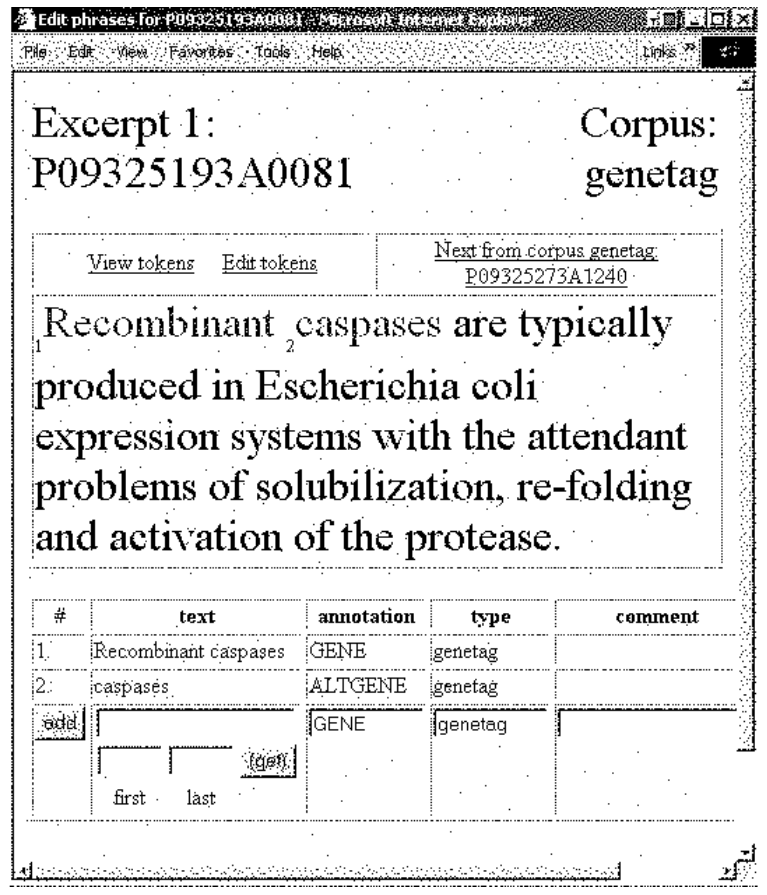

Figure 2: A screen capture of the annotator's interface and the GENETAG-05 annotations for a sentence.

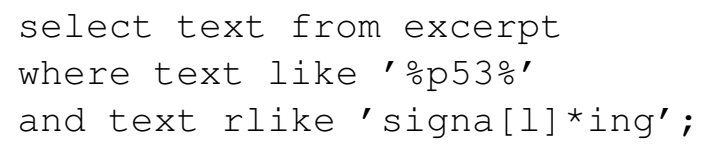

\subsection{Web Interface}

A web-based corpus editor was used to enter and review annotations. The code is being made available, as is, and requires that the data are loaded into a mysql database that can be accessed by a web server. The interface supports two annotation types: MedPost tags and arbitrary phrase annotations. MedPost tags are selectable from a pull-down menu of preprogrammed likely tags. For entering phrase annotations, the user highlights the desired phrase, and pressing the enter key computes and saves the first and last character offsets. The user can then enter the annotation code and an optional comment before saving it in the database. A screen dump of the phrase annotations for a sentence in the genetag corpus is shown in figure 2 .

The data from the database was dumped to the flat file format for this release. We have also included some files to accommodate previous users of the corpora. A perl program, alt_eval.perl is in- 
cluded that replaces the GENETAG evaluation program using non-whitespace character numbers instead of token numbers. Copies of the ABGene and MedPost corpora, in the original formats, are also included.

\section{Updates of Component Corpora}

\subsection{MedPost Update}

The MedPost corpus (Smith et. al., 2004) originally contained 5700 tokenized sentences. An additional 1000 annotated sentences have been added for this release. Each sentence in the MedPost corpus is fully tokenized, that is, divided into non-overlapping annotated portions, and each token is annotated with one of 60 part of speech tags (see Table 1). Minor corrections to the annotations have been made since the original release.

Since most of the original corpus, and all of the sentences used for training the MedPost tagger, were in the area of molecular biology, we added an additional 1000 sentences selected from random MEDLINE abstracts on the subject of clinical medicine. As a preliminary result, the trained MedPost tagger achieves approximately $96.9 \%$ accuracy, which is comparable to the $97.4 \%$ accuracy achieved on the subset of 1000 sentences selected randomly from all of MEDLINE. An example of a sentence from the clinical medicine collection is

$$
\begin{aligned}
& \text { Evidence }_{\mathrm{NN}} \quad \text { is }_{\mathrm{VBZ}} \quad \text { now }_{\mathrm{RR}} \text { available } \mathrm{JJ}_{\mathrm{J}} \\
& \text { to }_{\mathrm{TO}} \text { show }_{\mathrm{VVI}} \mathrm{a}_{\mathrm{DD}} \text { beneficial }{ }_{\mathrm{JJ}} \text { effect }_{\mathrm{NN}} \\
& \text { of }_{\text {II }} \text { bezafibrate }_{\mathrm{NN}} \text { on }_{\text {II }} \text { retarding }_{\mathrm{VVGN}} \\
& \text { atherosclerotic } \mathrm{JJ}_{\mathrm{JJ}} \text { processes }_{\mathrm{NNS}} \operatorname{and}_{\mathrm{CC}} \mathrm{in}_{\mathrm{II}} \\
& \text { reducing }_{\mathrm{VVGN}} \text { risk }_{\mathrm{NN}} \text { of }_{\mathrm{II}} \text { coronary }_{\mathrm{JJ}} \text { heart }_{\mathrm{NN}} \\
& \text { disease }_{\mathrm{NN}} \text {. }
\end{aligned}
$$

In addition to the token-level annotations, all of the gerunds in the MedPost corpus (these are tagged $V V G N$ ) were also examined and it was noted whether the gerund had an explicit subject, direct object, or adjective complement. This annotation is stored with an annotation of type gerund. To illustrate, the two gerunds in the previous example, retarding and reducing both have direct objects (retarding processes and reducing risk), and the gerund tag is entered as "o". The gerund annotations have been used to improve a noun phrase bracketer able to recognize gerundive phrases.

\subsection{GENETAG Update}

GENETAG is a corpus of MEDLINE sentences that have been annotated with gene and protein names. The closest related work is the GENIA corpus (Kim et. al., 2003). GENIA provides detailed coverage of a large number of semantic entities related to a specific subset of human molecular biology, whereas GENETAG provides gene and protein name annotations only, for a wide range of organisms and biomedical contexts (molecular biology, genetics, biochemistry, clinical medicine, etc.)

We are including a new version of GENETAG, GENETAG-05, as part of the MedTag system. GENETAG-05 differs from GENETAG in four ways: 1) the definition of a gene/protein entity has been modified, 2) significant annotation errors in GENETAG have been corrected, 3) the concept of a non-specific entity has been refined, and 4) character-based indices have been introduced to reduce tokenization problems. We believe that these changes result in a more accurate and robust corpus.

GENETAG-05 maintains a wide definition of a gene/protein entity including genes, proteins, domains, sites, sequences, and elements, but excluding plasmids and vectors. The specificity constraint requires that a gene/protein name must be included in the tagged entity. This constraint has been applied more consistently in GENETAG-05. Additionally, plain sequences like ATTGGCCTT$T A A C$ are no longer tagged, embedded names are tagged (ras-mediated), and significantly more terms have been judged to violate the specificity constraint (growth factor, proteases, protein kinase, ribonuclease, snoRNA, rRNA, tissue factor, tumor antigen, complement, hormone receptors, nuclear factors, etc.).

The original GENETAG corpus contains some entities that were erroneously tagged as gene/proteins. Many of these errors have been corrected in the updated corpus. Examples include camp-responsive elements, mu element, VDRE, melanin, dentin, myelin, auxin, BARBIE box, carotenoids, and cellulose. Error analysis resulted in the updated annotation conventions given in Table 1 .

Enzymes are a special class of proteins that catalyze biochemical reactions. Enzyme names have varying degrees of specificity, so the line drawn for 
tagging purposes is based on online resources ${ }^{1}$ as well as background knowledge. In general, tagged enzymes refer to more specific entities than untagged enzymes (tyrosine kinase vs. protein kinase, ATPase vs. protease). Enzymes that can refer to either DNA or RNA are tagged if the reference is specified (DNA endonuclease vs. endonuclease). Enzymes that do not require DNA/RNA distinction are tagged (lipase vs. ligase, cyclooxygenase vs. methylase). Non-specific enzymes are tagged if they clearly refer to a gene or protein, as in (1).

1) The structural gene for hydrogenase encodes a protein product of molecular mass $45820 \mathrm{Da}$.

Semantic constraints in GENETAG-05 are the same as those for GENETAG. To illustrate, the name in (2) requires rabies because $R I G$ implies that the gene mentioned in this sentence refers to the rabies immunoglobulin, and not just any immunoglobulin. In (3), the word receptor is necessary to differentiate $I G G$ receptor from $I G G$, a crucial biological distinction. In (4), the number 1 is needed to accurately describe a specific type of tumor necrosis factor, although tumor necrosis factor alone might be adequate in a different context.

2) rabies immunoglobulin (RIG)

3) IGG receptor

4) Tumor necrosis factor 1

Application of the semantic constraint can result in apparent inconsistencies in the corpus (immunoglobulin is sufficient on its own in some sentences in the corpus, but is insufficient in (2)). However, we believe it is important that the tagged entity retain its true meaning in the sentence context.

\section{Recommended Uses}

We have found the component corpora of MedTag to be useful for the following functions:

1) Training and evaluating part-of-speech taggers

2) Training and evaluating gene/protein named entity taggers

\footnotetext{
${ }^{1}$ http://cancerweb.ncl.ac.uk/omd/copyleft.html http://www.onelook.com/
}

3) Developing and evaluating a noun phrase bracketer for PubMed phrase indexing

4) Statistical analysis of grammatical usage in medical text

5) Feature generation for machine learning

The MedPost tagger was recently ported to Java and is currently being employed in MetaMap, a program that maps natural language text into the UMLS (Aronson,A.R., 2001).

\section{Conclusion}

We have merged three biomedical corpora into a collection of annotations called MedTag. MedTag uses a common relational database format along with a web interface to facilitate annotation consistency. We have identified the MEDLINE excerpts for each sentence and eliminated tokenization dependence, increasing the usability of the data. In GENETAG05 , we have clarified many grey areas for annotation, providing better guidelines for tagging these cases. For users of previous versions of the component corpora, we have included programs to convert from the new standardized format to the formats used in the older versions.

\section{References}

Aronson, A. R. 2001. Effective mapping of biomedical text to the UMLS Metathesaurus: the MetaMap program. Proc. AMIA Symp., 1721.

Kim, J.-D., Ohta, T., Tateisi, Y. and Tsujii, J. 2003. GENIA corpus: a semantically annotated corpus for biotextmining. Bioinformatics, 19: 180 - 182.

Tanabe, L and Wilbur, WJ. 2002. Tagging gene and protein names in biomedical text. Bioinformatics, 18, 1124-1132.

Tanabe L, Xie N, Thom, LH, Matten W, Wilbur, WJ: GENETAG: a tagged gene corpus for gene/protein named entity recognition. BMC Bioinformatics 2005.

Smith, L, Rindflesch, T, and Wilbur, WJ. 2004. MedPost: a part of speech tagger for biomedical text. Bioinformatics, 20(13) 2320-2321.

Yeh A, Hirschman L, Morgan A, Colosimo M: BioCreAtIvE task 1A: gene mention finding evaluation. BMC Bioinformatics 2005. 


\begin{tabular}{|c|c|c|c|c|}
\hline Entity Type & Problem & $\begin{array}{l}\text { GENETAG-05 } \\
\text { Convention }\end{array}$ & Positive Examples & $\begin{array}{l}\text { Negative } \\
\text { Examples }\end{array}$ \\
\hline $\begin{array}{l}\text { Protein } \\
\text { Families }\end{array}$ & $\begin{array}{l}\text { Some are named after } \\
\text { structural motifs. }\end{array}$ & $\begin{array}{l}\text { Do not tag } \\
\text { structures alone, } \\
\text { but tag structurally } \\
\text { related gene and } \\
\text { protein families. }\end{array}$ & $\begin{array}{l}\text { Zinc finger protein, } \\
\text { bZIP transcription } \\
\text { factor, homeobox } \\
\text { gene, TATA binding } \\
\text { protein }\end{array}$ & $\begin{array}{l}\text { Zinc finger, } \\
\text { helix-turn-helix } \\
\text { motif, leucine } \\
\text { zipper, homeobox, } \\
\text { TATA box }\end{array}$ \\
\hline Domains & $\begin{array}{l}\text { Name can refer to 1) the } \\
\text { amino acid content of a } \\
\text { sequence (PEST), 2) the } \\
\text { protein that binds the } \\
\text { sequence (TFIIIA DNA } \\
\text { binding domain), 3) a } \\
\text { homologous gene (SH2 - Src } \\
\text { homology domain 2), 4) the } \\
\text { first proteins in which the } \\
\text { domain was discovered (LIM, } \\
\text { PDZ), or 5) structural entities } \\
(P O Z \text {, zinc finger domain). }\end{array}$ & $\begin{array}{l}\text { Tag only if the } \\
\text { domain refers to a } \\
\text { gene or protein. } \\
\text { Immuno-globulin } \\
\text { regions are tagged. } \\
\text { (VH refers to the } \\
\text { Immuno-globulin } \\
\text { heavy chain } V \\
\text { region). }\end{array}$ & $\begin{array}{l}\text { BTB domain, LIM } \\
\text { domain, HECT } \\
\text { domain, VH } \\
\text { domain, SH2 } \\
\text { domain, TFIIIA } \\
\text { DNA binding } \\
\text { domain, } \\
\text { Krüppel-associated } \\
\text { box (KRAB) } \\
\text { domains, NF-IL6 } \\
\text { beta leucine zipper } \\
\text { domain } \\
\end{array}$ & $\begin{array}{l}\text { PEST domain, SR } \\
\text { domain, zinc finger } \\
\text { domain, b-Zip } \\
\text { domain, POZ } \\
\text { domain, GATA } \\
\text { domain, } R S \\
\text { domain, GAR } \\
\text { domain }\end{array}$ \\
\hline $\begin{array}{l}\text { Boxes, } \\
\text { Response } \\
\text { Elements and } \\
\text { Sites }\end{array}$ & $\begin{array}{l}\text { Name can refer to } 1) \text { the } \\
\text { sequence or site itself } \\
(T A A G), 2) \text { a non-protein that } \\
\text { binds to it (Glucocorticoid } \\
\text { Response Element), } 3 \text { ) a } \\
\text { protein that binds to it }(S p 1) \text {, } \\
\text { or } 4) \text { to homologous genes } \\
(V L 30) \text {. }\end{array}$ & $\begin{array}{l}\text { Tag only if the } \\
\text { sequence or site } \\
\text { refers to a gene or } \\
\text { protein. }\end{array}$ & $\begin{array}{l}\text { VL30 element, Zta } \\
\text { response elements, } \\
\text { activating protein } 1 \\
(A P-1) \text { site, Ets } \\
\text { binding site, } S P 1 \\
\text { site, } A P-2 \text { box }\end{array}$ & $\begin{array}{l}\text { GRE, TRE, cyclic } \\
\text { AMP response } \\
\text { element ( CRE), } \\
\text { TAAG sites, TGn } \\
\text { motif, TAR element, } \\
\text { UP element }\end{array}$ \\
\hline Hormones & Some are peptide hormones. & $\begin{array}{l}\text { Tag only peptide } \\
\text { hormones. }\end{array}$ & $\begin{array}{l}\text { Insulin, Glucagon, } \\
\text { growth hormone }\end{array}$ & $\begin{array}{l}\text { Estrogen, } \\
\text { Progesterone, } \\
\text { thyroid hormone }\end{array}$ \\
\hline $\begin{array}{l}\text { "and" } \\
\text { constructs }\end{array}$ & $\begin{array}{l}\text { Some conjuncts require the } \\
\text { entire construct. }\end{array}$ & $\begin{array}{l}\text { Unless both } \\
\text { conjuncts can stand } \\
\text { alone, tag them } \\
\text { together. }\end{array}$ & $\begin{array}{l}\text { TCR alpha and } \\
\text { beta, D-lactate and } \\
\text { D-glycerate } \\
\text { dehydrogenase }\end{array}$ & $\begin{array}{l}\text { TCR alpha, beta, } \\
\text { D-lactate, } \\
\text { D-glycerate } \\
\text { dehydrogenase }\end{array}$ \\
\hline $\begin{array}{l}\text { Viral } \\
\text { Sequences }\end{array}$ & $\begin{array}{l}\text { Promoters, enhancers, repeats } \\
\text { are distinguished by } \\
\text { organism. }\end{array}$ & $\begin{array}{l}\text { Tag only if the } \\
\text { organism is present. }\end{array}$ & $\begin{array}{l}\text { Viral LTR, HIV } \\
\text { long terminal } \\
\text { repeat, } S V 40 \\
\text { promoter }\end{array}$ & $\begin{array}{l}\text { LTR, long terminal } \\
\text { repeat }\end{array}$ \\
\hline Sequences & $\begin{array}{l}\text { Some sequences lack gene or } \\
\text { protein names. }\end{array}$ & $\begin{array}{l}\text { Tag only if a gene } \\
\text { name is included. }\end{array}$ & $\begin{array}{l}\text { NF kappa } B \\
\text { enhancer } \\
(T G G A A A T T C C)\end{array}$ & $\begin{array}{l}\text { TCTTAT, TTGGGG } \\
\text { repeats }\end{array}$ \\
\hline $\begin{array}{l}\text { Embedded } \\
\text { Names }\end{array}$ & $\begin{array}{l}\text { Some names are embedded in } \\
\text { non-gene text. }\end{array}$ & $\begin{array}{l}\text { Tag only the gene } \\
\text { part. }\end{array}$ & $\begin{array}{l}P \text {-47-deficient, } \\
\text { ras-transformed }\end{array}$ & $\begin{array}{l}P \text {-47-deficient, } \\
\text { ras-transformed }\end{array}$ \\
\hline $\begin{array}{l}\text { Transposons, } \\
\text { Satellites }\end{array}$ & Often repetitive sequences. & Tag if specific. & $\begin{array}{l}\text { L1 element, TN44, } \\
\text { copia } \\
\text { retrotransposon }\end{array}$ & $\begin{array}{l}\text { non-LTR } \\
\text { retrotransposon }\end{array}$ \\
\hline Antibodies & $\begin{array}{l}\text { Often use organism or disease } \\
\text { name. }\end{array}$ & Tag if specific. & $\begin{array}{l}\text { anti-SF group } \\
\text { rickettsiae }(S F G R)\end{array}$ & $\begin{array}{l}\text { antinuclear } \\
\text { antibody }\end{array}$ \\
\hline $\begin{array}{l}\text { Alternative } \\
\text { Transcripts }\end{array}$ & $\begin{array}{l}\text { Names differ from primary } \\
\text { transcript. }\end{array}$ & $\begin{array}{l}\text { Tag if primary } \\
\text { transcript named. }\end{array}$ & $\begin{array}{l}\text { I kappa } B \\
\text { gamma,VEGF20 }\end{array}$ & Exon 2, IIA \\
\hline
\end{tabular}

Table 2: Some problematic gene/protein annotations and conventions followed in GENETAG-05. 